\section{CRC: Operabilität von Lebermetastasen durch FOLFOXIRI plus Bevacizumab verbessert}

\author{
Beim Kolorektalkarzinom (CRC) mit nicht resezierbaren Metastasen ist \\ durch die Dreifachkombination FOLFOXIRI plus VEGF-Antikörper eine \\ kurative R0-Resektion oft doch noch möglich. Italienische Onkologen \\ überprüften das anhand gepoolter Studiendaten.
}

\begin{abstract}
E ür die Analyse wurden die Daten von F 11 zwischen 2010 und 2016 publizierten Studien ausgewertet. Fast alle der insgesamt fast 900 Patienten hatten jeweils zu Studienbeginn nicht resezierbare Metastasen eines CRC. Der Anteil der Patienten mit Metastasen ausschließlich in der Leber schwankte zwischen 18 und $100 \%$. Ermittelt wurde die Wirksamkeit der intensiven Dreifach-Chemotherapie FOLFOXIRI (Fluorouracil, Irinotecan, Oxaliplatin) in Kombination mit dem VEGF-Bevacizumab. In 6 Studien mit entsprechenden Angaben lag die mediane Zahl der verabreichten Zyklen zwischen 5-12. Primärer Endpunkt der Untersuchung war die Gesamtresektions-
\end{abstract}

rate, sekundäre Endpunkte die R0-Resektionsrate, die Gesamtansprechrate, das mediane progressionsfreie (PFS) und das Gesamtüberleben (OS).

Gesamt- sowie R0-Resektionsrate konnten jeweils in acht Studien ermittelt werden. Aus den gepoolten Daten errechneten sie eine Gesamtresektionsrate von $39,1 \%$ und eine R0-Resektionsrate von $28,1 \%$. Aus den Daten von 10 geeigneten Studien ergab sich eine Gesamtansprechrate von $69 \%$ (95\%-KI 65-72\%). Für die Ermittlung des OS standen Daten von 6 Studien zur Verfügung: Es betrug nach der intensiven Kombinationstherapie noch median 30,2 Monate. Schließlich geht aus der Analyse von 9
Studien hervor, dass durch diese Therapieform ein PFS von 12,4 Monaten erreicht wurde.

Nach Ansicht der Onkologen bestätigen die Ergebnisse der Untersuchung den klinischen Wert dieser intensiven Kombinationstherapie. Sie weisen darauf hin, dass mehr als $50 \%$ der Studienteilnehmer RAS- oder BRAF-mutierte Tumoren hatten. Deshalb sei bei Patienten ohne diese Mutationen ein noch besseres Ergebnis zu erwarten.

Fazit: Bei einem Teil der Patienten mit zunächst nicht resezierbaren Metastasen eines Kolorektalkarzinoms ist eine Operation der Metastasen sekundär doch noch möglich, wenn man sie mit einer intensiven chemotherapeutischen Dreifachkombination plus Bevacizumab behandelt. Fast 3 von 4 Patienten sprechen darauf an.

Peter Leiner

Tomasello G et al. FOLFOXIRI Plus Bevacizumab as Conversion Therapy for Patients With Initially Unresectable Metastatic Colorectal Cancer - A Systematic Review and Pooled Analysis. JAMA Oncol. 2017;3(7):e170278.

\section{Kolorektale Lebermetastasen: Erfolgreich resezieren auch nach Zweitlinien-Chemotherapie}

\author{
Patienten mit kolorektalen Lebermetastasen erhalten nach Versagen einer \\ neoadjuvanten Chemotherapie die Zweitlinien-Chemotherapie meist nur in \\ palliativer Intention. Jetzt liegen Daten zum Erfolg einer Resektion nach einer \\ Second-Line-Therapie vor.
}

\footnotetext{
E ine Leberresektion kommt für die - meisten Patienten mit kolorektalen Lebermetastasen (CLM) erst nach einer neoadjuvanten systemischen Chemotherapie in Frage. Deren Versagen wurde bisher als Prädiktor für ein schlechtes Ansprechen auf eine Zweitlinienbehandlung gewertet, und lediglich bei Progress wurde eine weitere systemische Therapie zur Krankheitskontrolle eingeleitet. Daten für den Erfolg einer präoperativen Second-Line-Behandlung mit anschließender CLM-Resektion gibt es kaum - obwohl ein solches Vorgehen die palliative in eine potenziell kurative Situation umkehren kann. Auf Basis einer großen internationalen, prospektiv geführten Ko-
}

horte konnten jetzt Überlebensdaten und Prädiktoren für den Erfolg von CLM-Patienten nach einer präoperativen SecondLine-Therapie ermittelt werden.

Die zwischen 2005 und 2013 beobachtete LiverMetSurvey-Kohorte umfasste 5.624 konsekutive Patienten mit einer Erstlinien- (Gruppe 1) und 791 konsekutive Patienten mit einer ZweitlinienChemotherapie (Gruppe 2). Nach einer medianen Nachbeobachtungszeit von 30,1 Monaten waren die Überlebensdaten beider Gruppen in etwa vergleichbar: Das mediane Gesamtüberleben (OS) sowie die 3-Jahres- und 5-Jahres-OS-Raten (OS) in Gruppe 2 lag bei 58,6 Monaten, 76 und $49 \%$ im Vergleich zu 58,9
Monaten, 71 und $49 \%$ in Gruppe $1(\mathrm{p}=$ 0,32 ). Die Zahlen für das krankheitsfreie Überleben (DFS) nach der Resektion waren allerdings in Gruppe 2 signifikant niedriger (17,2 Monate, 27 und $15 \%$ vs. 19,4 Monate, 32 und $23 \%, p=0,001$ ). Bei primär nicht resezierbaren Patienten war kein Unterschied beim OS und DFS nach Erst- oder Zweitlinie erkennbar. Als unabhängige Prädiktoren für ein schlechtes OS bei Zweitlinien-Patienten wurden u.a. positive primäre Lymphknoten, extrahepatische Metastasen, Progress während der Zweitlinie und eine R2-Resektion identifiziert.

Fazit: Eine Leberesektion bei Patienten mit CLM kann auch nach einer präoperativen Zweitlinien-Chemotherapie noch erfolgreich sein. Das OS ist vergleichbar mit dem von resezierten Patienten nach erfolgreicher Erstlinienbehandlung.

Barbara Kreutzkamp

Adam R et al. Resection of colorectal liver metastases after second-line chemotherapy: is it worthwile? A LiverMetSurvey analysis of 6415 patients. Eur J Cancer. 2017;78:7-15. 